\title{
CARACTERIZAÇÃO PÓS-COLHEITA DE SEIS CULTIVARES DE CARAMBOLA (Averrhoa carambola L.) ${ }^{1}$
}

\author{
GUSTAVO HENRIQUE DE ALMEIDA TEIXEIRA², JOSÉ FERNANDO DURIGAN², \\ LUIZ CARLOS DONADIO ${ }^{4}$ e JOSÉ ANTÔNIO ALBERTO DA SILVA ${ }^{5}$
}

\begin{abstract}
RESUMO - Avaliaram-se as qualidades física e química dos frutos de seis cultivares de carambola (Averrhoa carambola L.), propagadas pela Estação Experimental de Citricultura de Bebedouro, São Paulo, objetivando a obtenção de informações sobre a qualidade dos frutos produzidos nesta região. Foram utilizados frutos das cultivares Tean-ma, Fwang Tung, Arkin, Malásia, Golden Star e Nota-10. Os frutos foram analisados quanto à massa fresca, comprimento, largura, volume, porcentagem de suco, polpa e semente, número de sementes, coloração (L, Cromaticidade e ângulo Hue). Na polpa, determinou-se o pH, assim como o teor de umidade, sólidos solúveis totais (SST), acidez total titulável (ATT), vitamina C, açúcares solúveis totais e redutores, cinzas, proteína, pectina total e solúvel, e minerais $(\mathrm{Ca}, \mathrm{P}, \mathrm{K}, \mathrm{Mg}, \mathrm{S}, \mathrm{Cu}, \mathrm{Fe}, \mathrm{Mn}$ e $\mathrm{Zn})$. Os resultados foram avaliados estatisticamente através de um delineamento inteiramente casualizado e revelaram que os melhores frutos são os originários das cultivares Arkin, Tean-ma e Nota-10, pois são os maiores, os mais pesados e apresentam os maiores teores de sólidos solúveis totais (SST) e açúcares solúveis (AST), assim como boa relação SST/ATT. Os frutos da 'Arkin' mostraram coloração mais amarela, enquanto os da 'Golden Star' foram considerados de coloração mais clara, ou com maior translucidez. A textura foi menor nos frutos da 'Arkin', e se relacionou com a maior solubilização de suas pectinas.
\end{abstract}

Termos para indexação: melhoramento, qualidade, cultivares.

\section{POSTHARVEST CHARACTERIZATION OF SIX STAR FRUIT (Averrhoa carambola L.) GENOTYPES.}

ABSTRACT - Physical and chemical quality were valued of six carambola (Averrhoa carambola L.) cultivars, spreaded to the Bebedouro Experimenal Citrus Station, São Paulo, with purpose to obtainment informations about fruit quality in this region. Were utilized fruits of Tean-ma, Fwang Tung, Arkin, Malaysia, Golden Star and Nota-10 cultivars. The fruits were analysed to fresh weight, length, breadth, volume, percentage of juice, residue and seed, seed numbers, coloration ( $\mathrm{L}$, Chromaticity and Hue angle). In pulp, were determined $\mathrm{pH}$, as well as, the content of moisture, total soluble solids (TSS), total titratable acidity (TTA), vitamin C, total soluble sugars and reducings, ash, protein, total and soluble pectin, and minerals $(\mathrm{Ca}, \mathrm{P}, \mathrm{K}, \mathrm{Mg}, \mathrm{S}, \mathrm{Cu}, \mathrm{Fe}, \mathrm{Mn}$ and $\mathrm{Zn}$ ). The results were statiscaly valued according to completely randomized desing and showed Arkin, Tean-ma and Nota-10 cultivars, were the best fruits, because were larger, weighter and presented higher content of total soluble solids (TSS) and soluble sugars, as well as, good TSS/TTA ratio. Arkin fruits showed more yellow coloration while Golden Star's were considerate light, or more translucent. The texture was small in Arkin fruits an to linked with more pectin disasembly.

Index terms: Breeding, quality, cultivars.

\section{INTRODUÇÃO}

A caramboleira (Averrhoa carambola L.) pertence à família Oxalidaceae e tem como centro de origem o Sudeste asiático (Lennox \& Ragoonath, 1990; Nakasone \& Paul, 1998). A forma de seus frutos varia de oblongo a elipsóide, com 6 a $15 \mathrm{~cm}$ de comprimento e com 4 a 5 recortes longitudinais, que correspondem aos carpelos (Campbell \& Koch, 1989). A casca é translúcida, lisa e brilhante, e a cor varia do esbranquiçado ao amarelo-ouro intenso (Wilson, 1990).

Segundo Donadio et al. (2001), o início da seleção das variedades cultivadas de carambola ocorreu nas décadas de $30 \mathrm{e}$ 40 , sendo as variedades, até esta época, classificadas em dois grupos: ácidas e doces. Vários países asiáticos selecionaram a maioria das variedades atualmente cultivadas, todavia, na Flórida, também foram selecionados materiais como a cultivar Golden Star (Campbell \& Koch, 1989). Atualmente, as cultivares Arkin e B-10 são as de maior expressão, sendo a primeira predominante na Flórida (Campbell et al., 1989), e a segunda na Malásia (Lennox \& Ragoonath, 1990); entretanto, outras cultivares também têm sido plantadas, como a Golden Star, Fwang Tung e Maha.

No Brasil, principalmente no Estado de São Paulo, essa cultura está em franca expansão com pomares sendo formados com plantas "nativas" e/ou cultivares originárias da Flórida, cujas informações sobre desempenho, qualidade e composição química são escassas. Deste modo, este trabalho teve por objetivo caracterizar os frutos de seis cultivares de carambola introduzidas pela Faculdade de Ciências Agrárias e Veterinária da Universidade

\footnotetext{
1 (Trabalho 268/2000). Recebido: 13/12/2000. Aceito para publicação: 04/10/2001.

2 Aluno do curso de doutorado em agronomia, área de concentração produção vegetal da FCAV - UNESP Jaboticabal.

3 Prof. Adjunto do Departamento de Tecnologia, FCAV-UNESP. jfduri@fcav.unesp.br

4 Prof. Titular do Departamento de Horticultura, FCAV-UNESP. donadio@fcav.unesp.br

UNESP - Campus Jaboticabal, Via de acesso Prof. Paulo Donato Castelane, s/n, 14.870-000, Jaboticabal, SP, Brasil.

5 Eng. Agr., Dr., Estação Experimental de Citricultura de Bebedouro.
} 
Estadual Paulista (FCAV-UNESP), câmpus de Jaboticabal, e propagadas na Estação Experimental de Citricultura de Bebedouro (EECB) - São Paulo.

\section{MATERIAL E MÉTODOS}

Os frutos de seis cultivares, Tean-ma, Fwang Tung, Arkin, Malásia, Golden Star e Nota-10, esta última obtida de "seedlings" da cultivar B-10 selecionados na Estação Experimental de Citricultura de Bebedouro - São Paulo, foram colhidos no mês de julho de 2000. As plantas não foram desbastadas e, de cada material, foram colhidos 20 frutos no ponto típico e ideal para comercialização. No Laboratório de Tecnologia dos Produtos Agrícolas da FCAV / UNESP, câmpus de Jaboticabal, os frutos foram divididos em 6 tratamentos, correspondentes às cultivares, com 4 repetições de 5 frutos cada. Esse planejamento permitiu analisar os resultados estatisticamente, conforme um delineamento inteiramente casualizado.

A massa fresca foi determinada através de pesagem em balança semi-analítica com precisão de 0,01 grama. O comprimento e a largura foram determinados através de paquímetro Maub, sendo as leituras tomadas longitudinalmente entre as cicatrizes do pedúnculo - estigma, e no sentido equatorial, respectivamente. $O$ volume foi determinado através da imersão dos frutos em béquer graduado. Para a determinação da textura, foi utilizado um penetrômetro Bishop mod. FT 327 com ponteira de $8 \mathrm{~mm}$, fazendose duas leituras por fruto, na região carpelar. A coloração foi determinada utilizando-se um reflectômetro Minolta CR-200b, segundo o sistema proposto pela "Commission Internacional de L'eclaraige" (CIE). Para esta análise, foram feitas duas leituras por fruto, na região carpelar, sendo a coloração expressa pela luminosidade ( $\left.\mathrm{L}^{*}\right)$, cromaticidade (Croma) e pelo ângulo Hue, conforme metodologia descrita por Miller \& McDonald (1998). A determinação do rendimento em suco, sementes e resíduo foi feita, utilizando-se de uma centrífuga doméstica Arno para a separação dessas frações, as quais foram pesadas separadamente. Após a pesagem, as sementes foram contadas, determinando-se o número médio por fruto.

No suco, foram determinados os teores de sólidos solúveis totais (SST), acidez total titulável (ATT), assim como o pH (A.O.A.C., 1975), o teor de ácido ascórbico pelo método de Tillman (Strohecker \& Henning, 1967), e calculou-se a relação SST/ATT. Dos frutos analisados, tomou-se uma porção de polpa triturada, que foi mantida congelada a $-10^{\circ} \mathrm{C}$ até serem determinados os teores de umidade, cinzas e proteína (A.O.A.C., 1975); açúcares solúveis totais (Yemn \& Willis, 1954); açúcares redutores (Miller, 1959); pectina total e solúvel, cujas frações foram extraídas pelo método de McCready \& McComb (1952) e dosadas através da reação de condensação com m-hidroxidifenil (Blumenkrantz \& Asboe-Hansen, (1973) e minerais (Sarruge \& Haag, 1974).

\section{RESULTADOS E DISCUSSÃO}

Na Tabela 1, são apresentadas as características físicas dos frutos das seis cultivares estudadas. Quanto à massa fresca, os frutos da cultivar Nota-10 apresentaram os maiores valores, que não diferiram das cultivares Arkin, Malásia e Tean-ma, enquanto os da 'Fwang Tung' e 'Golden Star' mostraram-se como os menos pesados, sendo este último o de menor massa. Esses resultados estão de acordo com os de Lennox \& Ragoonath (1990), que relataram ser a cultivar B-10 produtora de frutos pesados (100 a 300g) e a Arkin de frutos médios (90 a 200g). A cultivar Fwang Tung foi considerada como sendo produtora de frutos pesados, o que não se repetiu neste trabalho, possivelmente devido à falta de desbaste dos frutos. Quanto ao comprimento, a 'Arkin' apresentou os maiores valores $(\mathrm{P}<0,05)$, seguida da 'Malásia', 'Fwang Tung', Nota-10, 'Tean-ma' e 'Golden Star'. A largura também foi maior na 'Arkin', com os frutos da 'Tean-ma', 'Fwang Tung' e Nota-10 não diferindo estatisticamente dela, seguidos da 'Golden Star' e da 'Malásia', que apresentaram as menores larguras. Estes valores permitem afirmar que os frutos das cultivares Arkin, Fwang Tung e Nota10 são os de maior tamanho. Os da 'Malásia', apesar do bom comprimento $(11,11 \mathrm{~cm})$, apresentaram largura menor, sendo, portanto, mais finos que os demais, e os da 'Golden Star', os menores. Campbell \& Koch (1989) relataram variações no comprimento $(10,1 \mathrm{a} 7,6 \mathrm{~cm})$ e no diâmetro $(6,3$ a $4,7 \mathrm{~cm})$ dos frutos da 'Golden Star', colhidos no início e final da safra, respectivamente. Além dessa grande variação, nas árvores, existem frutos com diferentes idades, o que dificulta a escolha dos frutos a serem colhidos. De modo geral, a massa fresca e o tamanho relacionaram-se com o volume dos frutos, o qual foi maior nas cultivares Arkin, Nota-10 e Malásia, com a Fwang Tung e Golden Star apresentando os menores volumes.

Pode-se observar ainda, na Tabela 1, que não houve diferença significativa quanto aos teores de suco e de resíduo, enquanto a porcentagem de sementes variou de $0,51 \%$ a $2,90 \%$, com a 'Golden Star' e 'Malásia' apresentando as maiores porcentagens $(2,25 \%$ e 2,90\%), seguidas 'Fwang Tung' e 'Teanma' $(1,63 \%$ e $1,11 \%)$, e com a 'Arkin' e a Nota-10 apresentando as menores $(0,51 \%$ e $0,65 \%)$. A menor porcentagem nessas cultivares é devida ao pequeno número de sementes, 3,88 e 7,88 para a Nota-10 e para a 'Arkin", respectivamente.

Quanto à textura, os frutos da 'Fwang Tung' mostraramse mais firmes $(\mathrm{P}<0,05)$, seguidos da 'Malásia' e da Nota-10, que não diferiram entre si, e da 'Tean-ma' e 'Golden Star'. Os frutos da 'Arkin' mostraram o menor valor para esta variável, 48,61 N, possivelmente devido ao estádio fisiológico mais adiantado. Entretanto, os valores encontrados para a textura, de todas as cultivares, foram maiores do que os observados por Miller \& McDonald (1998), que relataram valores de 24,3 $\mathrm{N}$ para os frutos maduros da cultivar Arkin.

Os resultados de coloração (Tabela 1) mostram que os frutos das cultivares Fwang Tung e Golden Star têm maior luminosidade (L) e a Nota-10, a menor. O ângulo Hue foi maior para os frutos da 'Fwang Tung', seguidos pelos da Nota-10, e da 'Golden Star' e 'Tean-ma'. Este ângulo foi menor para os da cultivar Arkin $(99,97)$, ou seja, estavam com cor mais amarela que os demais, cujos valores mostram que estes se apresentavam mais verdes, destacadamente os da 'Fwang Tung' $(115,04)$. Os valores de cromaticidade (Croma) mais altos da 'Fwang Tung' e 'Malásia' reafirmam sua coloração verde mais intensa, assim como as da 'Arkin', a coloração amarela intensa. Os frutos da 'Fwang Tung' e 'Golden Star' mostraram-se com coloração considerada 
clara, principalmente os da 'Golden Star', cujos valores de L e Croma reforçam sua translucidez.

O grande conteúdo de umidade na polpa da carambola, já relatado por Wilson (1990), também foi observado neste trabalho, conforme os resultados apresentados na Tabela 2. Não houve diferença significativa entre cultivares, e a variação de $87,81 \%$ a $90,69 \%$ está de acordo com o observado por Lennox \& Ragoonath (1990). Quanto aos conteúdos de sólidos solúveis totais (SST) no suco, as cultivares Arkin e Malásia apresentaram maior Brix, 10,25 e $10,13{ }^{\circ}$ Brix, respectivamente, seguidos da Nota10, e a 'Fwang Tung' e 'Tean-ma', que não diferiram entre si. A 'Golden Star' apresentou os menores teores, 7,88 ${ }^{\circ} \mathrm{Brix}$, os quais são semelhantes ao relatado por Lennox \& Ragoonath (1990). A acidez total titulável (ATT) foi maior nos frutos da 'Malásia' $(P<0,05)$, com valores decrescentes entre as cultivares Fwang Tung, Nota-10 e Golden Star, que não diferiram entre si, com os menores teores nos frutos da 'Arkin' e 'Tean-ma'. O maior teor de SST e a menor ATT fez com que os frutos da 'Arkin' e da Nota-10 apresentassem as maiores relações de SST/ATT, sendo, portanto, os mais doces, e a 'Malásia', apesar do bom teor de SST, o menor (Tabela 2). Segundo Wilson (1990), a relação SST/ ATT ótima para o consumo da carambola é de 12,6 (8,6\% de SST e $0,69 \%$ de ATT), o que é menor que os valores observados para todas as cultivares estudadas neste trabalho, indicando que os frutos produzidos em Bebedouro, São Paulo, se mostraram mais "doces". Não se observaram diferenças significativas entre os valores de $\mathrm{pH}$ do suco, cuja variação foi de 3,35 a 3,60. Estes valores são menores que o observado por Miller \& McDonald (1998), 4,2, mas estão dentro da variação de 2,9 a 4,2 relatada por Wilson (1990). Quanto aos teores de ácido ascórbico, as cultivares Malásia e Arkin apresentaram os maiores valores, 26,28 e 23,99 mg de ácido ascórbico. $100 \mathrm{~g}^{-1}$, seguidos da 'Golden Star', 'Fwang
Tung', 'Nota-10' e 'Tean-ma', que mostraram os menores valores, $12,54 \mathrm{mg}$ de ácido ascórbico. $100 \mathrm{~g}^{-1}$. Estes resultados são semelhantes aos de Wilson (1990), mas inferiores dos relatados por Lennox \& Ragoonath (1990).

Ainda na Tabela 2, pode-se observar que os teores de açúcares solúveis totais (AST) foram maiores nos frutos da 'Arkin' ( $\mathrm{P}<0,05)$, que corresponderam, em média, a $84,68 \%$ do conteúdo de SST. Estes teores não diferiram dos apresentados pela 'Fwang Tung', 'Nota-10' e 'Malásia', e foram superiores aos da 'Teanma' e 'Golden Star', que apresentaram os menores teores. Quanto aos teores de açúcares redutores (AR), os frutos da 'Fwang Tung' e 'Arkin' apresentaram os maiores teores $(\mathrm{P}<0,05)$, enquanto os da 'Golden Star', os menores. Os resultados dos conteúdos de açúcares, tanto AST quanto AR, reforçam o observado quanto ao sabor mais doce dos frutos das cultivares Arkin, Fwang Tung, Tean-ma e Nota-10. Estes resultados estão de acordo com o observado por Campbell \& Koch (1989), ou seja, que os frutos da cultivar Arkin têm maior teor de glicose e frutose (7,5 e 8,8 mg.g $\left.{ }^{-1}\right)$ que os da 'Golden Star' (6,0 e 6,9 mg.g${ }^{1}$ ), o que reafirma a classificação da Arkin como mais "doce". Os demais parâmetros analisados, cinzas, proteína, pectina total e solúvel, não apresentaram diferenças significativas entre as cultivares (Tabela 2). Os teores médios de cinzas $(0,33 \%)$ e proteína (0,66\%) estão de acordo com o relatado por Taylor (1993) e Lennox \& Ragoonath (1990), e os resultados de pectina, apesar de não variarem entre as cultivares, apresentaram diferenças na sua porcentagem de solubilização, com a 'Arkin' apresentando a maior solubilização (19,23\%), seguida da 'Tean-ma' $(14,35 \%)$ e das demais, 'Malásia (10,15\%), 'Golden Star' (8,97\%), B-1-(8,26\%) e 'Fwang Tung' (6,75\%). A maior porcentagem de solubilização das pectinas da 'Arkin' e sua textura mais frágil (Tabela 1) sugerem que os frutos desta cultivar poderiam estar

TABELA 1 - Características físicas dos frutos de seis cultivares de carambola (Averrhoa carambola L).

\begin{tabular}{|c|c|c|c|c|c|c|c|c|c|c|c|c|}
\hline \multirow{3}{*}{ CULTIVAR } & \multicolumn{12}{|c|}{ PARÂMETROS } \\
\hline & \multirow{2}{*}{$\begin{array}{c}\text { Massa fresca } \\
(\mathrm{g})\end{array}$} & \multirow{2}{*}{$\begin{array}{l}\text { Comprimento } \\
(\mathrm{cm})\end{array}$} & \multirow{2}{*}{$\begin{array}{l}\text { Largura } \\
(\mathrm{cm})\end{array}$} & \multirow{2}{*}{$\begin{array}{c}\text { Volume } \\
(\mathrm{ml})\end{array}$} & \multirow{2}{*}{$\begin{array}{c}\text { Suco } \\
(\%)\end{array}$} & \multirow{2}{*}{$\begin{array}{c}\text { Resíduo } \\
(\%)\end{array}$} & \multirow{2}{*}{$\begin{array}{c}\text { Semente } \\
(\%)\end{array}$} & \multirow{2}{*}{$\begin{array}{l}\text { Semente } \\
\text { (un) }\end{array}$} & \multirow{2}{*}{$\begin{array}{l}\text { Textura } \\
(\mathrm{N})\end{array}$} & \multicolumn{3}{|c|}{ Coloração } \\
\hline & & & & & & & & & & $\mathrm{L}$ & Croma & Hue \\
\hline Tean-ma & $116,15 \mathrm{abc}$ & $10,38 \mathrm{c}$ & $6,36 \mathrm{ab}$ & $111,25 \mathrm{ab}$ & 57,61 & 41,29 & $1,11 \mathrm{~cd}$ & $13,13 \mathrm{a}$ & $56,55 \mathrm{~b}$ & $46,54 \mathrm{~b}$ & $21,99 \mathrm{~b}$ & $107,11 \mathrm{c}$ \\
\hline Fwang Tung & $96,43 \mathrm{~cd}$ & $11,01 \mathrm{abc}$ & $6,43 \mathrm{ab}$ & $86,00 \mathrm{~b}$ & 57,54 & 40,84 & $1,63 \mathrm{bc}$ & $13,38 \mathrm{a}$ & $63,70 \mathrm{a}$ & $52,36 \mathrm{a}$ & 25,89 a & $115,04 \mathrm{a}$ \\
\hline Arkin & $131,74 \mathrm{ab}$ & $11,36 \mathrm{a}$ & $6,82 \mathrm{a}$ & $128,75 \mathrm{a}$ & 53,25 & 46,25 & $0,51 \mathrm{~d}$ & $7,88 \mathrm{~b}$ & $48,61 \mathrm{c}$ & $44,57 \mathrm{bc}$ & $24,03 \mathrm{ab}$ & $99,97 \mathrm{~d}$ \\
\hline Malásia & $111,74 \mathrm{bc}$ & $11,11 \mathrm{ab}$ & $5,58 \mathrm{c}$ & $122,50 \mathrm{a}$ & 53,63 & 44,12 & $2,25 \mathrm{ab}$ & $14,25 \mathrm{a}$ & $60,56 \mathrm{ab}$ & $46,62 \mathrm{~b}$ & $24,93 \mathrm{a}$ & $110,94 \mathrm{bc}$ \\
\hline Nota -10 & $135,81 \mathrm{a}$ & $10,63 \mathrm{bc}$ & $6,28 a b$ & $125,25 \mathrm{a}$ & 55,34 & 44,02 & $0,65 \mathrm{~d}$ & $3,88 \mathrm{~b}$ & $62,33 \mathrm{ab}$ & $43,78 \mathrm{c}$ & $22,24 \mathrm{~b}$ & $111,16 \mathrm{ab}$ \\
\hline Golden Star & $89,07 \mathrm{~d}$ & $9,41 \mathrm{~d}$ & $6,07 \mathrm{bc}$ & $95,00 \mathrm{~b}$ & 52,34 & 44,77 & $2,90 \mathrm{a}$ & $16,63 \mathrm{a}$ & $57,04 \mathrm{~b}$ & $50,56 \mathrm{a}$ & $17,46 \mathrm{c}$ & $110,62 \mathrm{bc}$ \\
\hline $\mathrm{CV}$ & $21,61 \%$ & $7,27 \%$ & $11,07 \%$ & $25,25 \%$ & $11,00 \%$ & $13,62 \%$ & $27,63 \%$ & $18,94 \%$ & $8,92 \%$ & $5,52 \%$ & $12,70 \%$ & $4,02 \%$ \\
\hline
\end{tabular}

- Nas colunas, médias seguidas pela mesma letras não diferem estatisticamente entre si, pelo teste de Tukey $(\mathrm{P}<0,05)$.

TABELA 2 - Características químicas e físico-químicas dos frutos de seis cultivares de carambola (Averrhoa carambola L).

\begin{tabular}{|c|c|c|c|c|c|c|c|c|c|c|c|c|}
\hline \multirow[b]{2}{*}{ CULTIVAR } & \multicolumn{12}{|c|}{ PARÂMETROS } \\
\hline & $\begin{array}{c}\text { Umidade } \\
(\%)\end{array}$ & $\begin{array}{c}\text { SST } \\
\left({ }^{0} \text { Brix }\right)\end{array}$ & $\begin{array}{l}\text { ATT } \\
(\%)\end{array}$ & SST/ATT & $\mathrm{pH}$ & $\begin{array}{c}\text { AA } \\
\left(\mathrm{mg} \cdot 100 \mathrm{~g}^{-1}\right)\end{array}$ & $\begin{array}{l}\text { AST } \\
(\%)\end{array}$ & $\begin{array}{l}\mathrm{AR} \\
(\%)\end{array}$ & $\begin{array}{c}\text { Cinzas } \\
(\%)\end{array}$ & $\begin{array}{c}\text { Proteína } \\
(\%)\end{array}$ & $\begin{array}{l}\text { Pectina Total } \\
\left(\mathrm{mg} .100 \mathrm{~g}^{-1}\right)\end{array}$ & 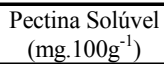 \\
\hline Tean-ma & 90,66 & $9,0 \mathrm{ab}$ & $0,37 \mathrm{c}$ & $24,62 \mathrm{ab}$ & 3,51 & $12,54 \mathrm{~d}$ & $7,30 \mathrm{bc}$ & $6,87 \mathrm{bc}$ & 0,26 & 0,61 & 242,86 & 35,10 \\
\hline Fwang Tung & 87,81 & $9,25 \mathrm{ab}$ & $0,53 \mathrm{ab}$ & $17,72 \mathrm{bc}$ & 3,40 & $17,09 \mathrm{~cd}$ & $8,53 \mathrm{ab}$ & $8,39 \mathrm{a}$ & 0,38 & 0,83 & 306,99 & 20,52 \\
\hline Arkin & 88,44 & $10,25 \mathrm{a}$ & $0,37 \mathrm{c}$ & $27,83 \mathrm{a}$ & 3,60 & $23,99 \mathrm{ab}$ & $8,68 \mathrm{a}$ & $7,98 \mathrm{a}$ & 0,46 & 0,67 & 193,75 & 32,11 \\
\hline Malásia & 91,13 & $10,13 \mathrm{a}$ & $0,63 \mathrm{a}$ & $16,03 \mathrm{c}$ & 3,35 & $26,28 \mathrm{a}$ & $8,30 a b$ & $7,56 \mathrm{ab}$ & 0,27 & 0,63 & 324,12 & 31,65 \\
\hline Nota -10 & 89,03 & $9,25 \mathrm{ab}$ & $0,43 \mathrm{bc}$ & $22,38 \mathrm{abc}$ & 3,38 & $18,14 \mathrm{c}$ & $8,48 a b$ & $7,32 \mathrm{ab}$ & 0,28 & 0,62 & 333,86 & 27,57 \\
\hline Golden Star & 90,69 & $7,88 \mathrm{~b}$ & $0,43 \mathrm{bc}$ & $18,69 \mathrm{bc}$ & 3,51 & $19,85 \mathrm{bc}$ & $6,77 \mathrm{c}$ & $6,10 \mathrm{c}$ & 0,35 & 0,60 & 343,72 & 29,84 \\
\hline $\mathbf{C V}$ & $2,40 \%$ & $7,45 \%$ & $12,93 \%$ & $16,43 \%$ & $4,56 \%$ & $10,89 \%$ & $6,93 \%$ & $6,10 \%$ & $29,07 \%$ & $28,47 \%$ & $25,75 \%$ & $27,98 \%$ \\
\hline
\end{tabular}

- SST - sólidos solúveis totais, ATT - acidez total titulável, AA - ácido ascórbico, AST - açúcares solúveis totais, AR - açúcares redutores solúveis.

- Nas colunas, médias seguidas pela mesma letras não diferem estatisticamente entre si, pelo teste de Tukey $(\mathrm{P}<0,05)$. 
TABELA 3 - Teor de minerais na polpa fresca dos frutos seis cultivares de carambola (Averrhoa carambola L).

\begin{tabular}{|c|c|c|c|c|c|c|c|c|c|}
\hline \multirow[b]{2}{*}{ CULTIVAR } & \multicolumn{9}{|c|}{ MINERAIS } \\
\hline & $\begin{array}{c}\text { Cálcio } \\
\left(\mathrm{mg} \cdot 100 \mathrm{~g}^{-1}\right)\end{array}$ & $\begin{array}{c}\text { Fósforo } \\
\left(\mathrm{mg} .100 \mathrm{~g}^{-1}\right)\end{array}$ & $\begin{array}{c}\text { Potássio } \\
\left(\mathrm{mg} .100 \mathrm{~g}^{-1}\right)\end{array}$ & $\begin{array}{l}\text { Magnésio } \\
\left(\mathrm{mg} .100 \mathrm{~g}^{-1}\right)\end{array}$ & $\begin{array}{c}\text { Enxofre } \\
\left(\mathrm{mg} .100 \mathrm{~g}^{-1}\right)\end{array}$ & $\begin{array}{c}\text { Cobre } \\
\left(\mathrm{mg} .100 \mathrm{~g}^{-1}\right)\end{array}$ & $\begin{array}{c}\text { Ferro } \\
\left(\mathrm{mg} .100 \mathrm{~g}^{-1}\right)\end{array}$ & $\begin{array}{c}\text { Manganês } \\
\left(\mathrm{mg} .100 \mathrm{~g}^{-1}\right)\end{array}$ & $\begin{array}{c}\text { Zinco } \\
\left(\mathrm{mg} \cdot 100 \mathrm{~g}^{-1}\right) \\
\end{array}$ \\
\hline Tean-ma & 4,35 & $6,77 \mathrm{~b}$ & 84,56 & 5,97 & 3,04 & 0,14 & $0,57 \mathrm{~b}$ & $0,42 \mathrm{ab}$ & 0,23 \\
\hline Fwang Tung & 4,43 & $13,05 \mathrm{a}$ & 136,25 & 9,06 & 6,58 & 0,22 & $0,96 \mathrm{ab}$ & $0,25 \mathrm{~b}$ & 0,38 \\
\hline Arkim & 4,73 & $11,47 \mathrm{ab}$ & 132,95 & 7,29 & 6,12 & 0,16 & $0,86 \mathrm{ab}$ & $0,25 \mathrm{~b}$ & 0,36 \\
\hline Malásia & 3,64 & $7,72 a b$ & 99,93 & 5,71 & 5,30 & 0,17 & $1,06 \mathrm{ab}$ & $0,16 \mathrm{~b}$ & 0,30 \\
\hline Nota -10 & 4,43 & $9,75 \mathrm{ab}$ & 107,56 & 7,73 & 5,16 & 0,18 & $1,35 \mathrm{a}$ & $0,23 \mathrm{~b}$ & 0,26 \\
\hline Golden Star & 4,61 & $10,93 \mathrm{ab}$ & 135,53 & 8,91 & 4,49 & 0,15 & $0,77 \mathrm{ab}$ & $0,61 \mathrm{a}$ & 0,30 \\
\hline $\mathrm{CV}$ & $20,55 \%$ & $23,95 \%$ & $30,34 \%$ & $26,11 \%$ & $30,98 \%$ & $28,41 \%$ & $29,35 \%$ & $38,99 \%$ & $32,52 \%$ \\
\hline
\end{tabular}

- Nas colunas, médias seguidas pela mesma letras não diferem estatisticamente entre si, pelo teste de Tukey (P<0,05).

fisiologicamente mais adiantados por ocasião da colheita, apesar da aparência adequada, pois sabe-se que, durante o amadurecimento, há hidrólise das pectinas da parede celular e amaciamento dos frutos (Huber, 1983; Tucker, 1993).

$\mathrm{O}$ teor de minerais na polpa fresca das frutos das seis cultivares estudadas é apresentado na Tabela 3. Pode-se observar que não houve diferença significativa para o cálcio, potássio, magnésio, enxofre, cobre e zinco. O maior teor de fósforo foi verificado nos frutos da 'Fwang Tung' $(\mathrm{P}<0,05)$ e o menor, nos da 'Tean-ma', com os demais não diferindo entre si e dessas duas cultivares. O potássio é o mineral em maior quantidade na polpa da carambola $\left(116,13 \mathrm{mg} \cdot 100 \mathrm{~g}^{-1}\right.$ de polpa fresca), seguido do fósforo $\left(9,95 \mathrm{mg} .100 \mathrm{~g}^{-1}\right.$ de polpa fresca). Estes resultados estão de acordo com o relatado por Wilson (1990), que observou teores de $110 \mathrm{mg} .100 \mathrm{~g}^{-1}$ e $11 \mathrm{mg} .100 \mathrm{~g}^{-1}$ para estes minerais, respectivamente. Dos micronutrientes, somente o ferro e o manganês apresentaram diferenças significativas entre as cultivares, com a Nota-10 apresentando os maiores teores de ferro e a 'Tean-ma' os menores, e a 'Golden Star' e 'Tean-ma', os maiores de manganês.

\section{CONCLUSÕES}

Pelos resultados apresentados, pode-se afirmar que os melhores frutos são originários das cultivares Arkin, Tean-ma e Nota-10, pois são os maiores, os mais pesados e apresentam os maiores teores de sólidos solúveis totais (SST) e açúcares solúveis (AST), assim como boa relação SST/ATT. Os frutos da 'Arkin' mostraram coloração mais amarela, enquanto os da 'Golden Star' foram considerados de coloração mais pobre, ou com maior translucidez. A textura foi menor nos frutos da 'Arkin', e relacionou-se com a maior solubilização de suas pectinas.

\section{REFERÊNCIAS BIBLIOGRÁFICAS}

ASSOCIATION OF OFFICIAL ANALYTICAL CHEMISTS. Official methods of analysis of the association of official analytical chemists. 12.ed. Washington: A.O.A.C,. 1975. 1115p.

BLUMENKRANTZ, N.; ASBOE-HANSEN, G. New method for quantitative determination of uronics acids. Analytical Biochemistry, New York, v.54, p.484-9, 1973.

CAMPBELL, C. A.; HUBER, D. J.; KOCH, K. E. Postharvest changes in sugar, acids, and color of carambola fruit at various temperatures. HortScience, Alexandria, v.24, n.3, p.472-5, 1989.

CAMPBELL, C. A.; KOCH, K. E. Sugar/acid composition and develompment of sweet and tart carambola fruit. Journal of American Society for Horticultural Science, Alexandria, v. 114, n.3, p.455-7, 1989.

DONADIO, L. C.; SILVA, J. A A.; ARAÚJO, P. S.; PRADO, R. M. Caramboleira (Averrhoa carambola L.). Jaboticabal: Sociedade Brasileira de Fruticultura, 2001. 81p. (Série Frutas Potenciais).

HUBER, D. J. The role of cell wall hydrolases in fruits softening. Horticultural Reviews, Alexandria, v.5, p.169-219, 1983.

LENNOX, A.; RAGOONATH, J. Carambola and bilimbi. Fruits, Paris, v.45, n.5, p.497-501, 1990.

McCREADY, P. M.; McCOMB, E. A. Extraction and determination of total pectin materials. Analytical Chemistry, Washington, v.24, n.12, p.1586-8, 1952.

MILLER, G. L. Use of dinitrosalicylic acid reagent for determination of reducing sugars. Analytical Chemistry, Washington, v.31, n.3, p.426-8, 1959.

MILLER, W. R.; McDONALD, R. E. Reducing irradiation damange to 'Arkin' carambola by plastic pachaging or storage temperature. HortScience, Alexandria, v.33, n.6, p.1038-41, 1998.

NAKASONE, H. K.; PAULL, R. E. Tropical fruits. Wallingford: CAB International, 1998. p.132-148.

SARRUGE, J. R.; HAAG, H. P. Análises químicas em plantas. Piracicaba: ESALQ-USP, 1974. 56p.

STROHECKER, R.L.; HENNING, H.M. Analisis de vitaminas: métodos comprobados. Madrid: Paz Montalvo, 1967. 428p.

TAYLOR, J. E. Exotics. In: SEYMOUR, G. B.; TAYLOR, J. E.; TUCKER, G. A. Biochemistry of fruit ripening. Cambridge: Chapman \& Hall, 1993.p.151-187.

TUCKER, G. A. Introduction. In: SEYMOUR, G. B., TAYLOR, J. E., TUCKER, G. A. Biochemistry of fruit ripening. Cambridge: Chapman \& Hall, 1993. p.3-43. 
YEMN, E. W.; WILLIS, A. J. The estimation of carbohydrate in plant extracts by anthrone. The Biochemical Journal, London, v.57, n.2, p.508-14, 1954.
WILSON, C. W. III. Carambola and Bilinbi. In: NAGY, S.; SHAW, P. E.; WARDOWSKY, F. S. Fruits of tropical and subtropical origem: composition, properties and uses. Lake Alfred, Florida: Florida Science Source, 1990. p.277-301. 